TRANSACTIONS OF THE

AMERICAN MATHEMATICAL SOCIETY

Volume 364, Number 7, July 2012, Pages 3861-3873

S 0002-9947(2012)05578-4

Article electronically published on January 25, 2012

\title{
SPLITTINGS AND THE ASYMPTOTIC TOPOLOGY OF THE LAMPLIGHTER GROUP
}

\author{
PANOS PAPASOGLU
}

\begin{abstract}
It is known that splittings of one-ended finitely presented groups over 2-ended groups can be characterized geometrically. Here we show that this characterization does not extend to all finitely generated groups, by showing that the lamplighter group is coarsely separated by quasi-lines.

It is also known that virtual surface groups are characterized in the class of one-ended finitely presented groups by the property that their Cayley graphs are coarsely separated by quasi-circles. Answering a question of Kleiner we show that the Cayley graph of the lamplighter group is coarsely separated by quasi-circles. It follows that the quasi-circle characterization of virtual surface groups does not extend to the finitely generated case.
\end{abstract}

\section{INTRODUCTION}

Stallings 14 showed that a finitely generated group has more than one end if and only if it splits over a finite group. This gives a geometric characterization of finitely generated groups which split over a finite group, where here geometric means in the sense of quasi-isometries.

One has a similar geometric characterization for splittings over 2-ended groups which applies to finitely presented groups. It was shown in [12] (see also [10]) that a one-ended finitely presented group, which is not virtually a surface group, splits over a 2-ended group if and only if its Cayley graph is coarsely separated by a quasi-line. Another geometric characterization of splittings of hyperbolic groups over 2-ended groups was given earlier by Bowditch [1. It is natural to ask whether the characterization given in 12 applies in fact to all one-ended finitely generated groups (as it is the case for Stallings' theorem).

Here we show that there is a one-ended finitely generated group (the lamplighter group) which is coarsely separated by a quasi-line but does not split over a 2-ended group. It turns out that the same group can be used to answer a question of Kleiner (13, problem 4.5).

We would like to thank D. Vavrichek, V. Metaftsis and the anonymous referee for suggestions that improved the exposition of this paper.

\section{Preliminaries}

2.1. Asymptotic topology. We recall some definitions relating to asymptotic topology. The idea (due to Gromov) behind these definitions is that one can develop a large scale analog of topological notions. It is in a similar vein to large scale geometry, except that in asymptotic topology one tries to give a 'rough' or 'large

Received by the editors September 19, 2010 and, in revised form, December 30, 2010, February 1, 2011, and February 4, 2011.

2010 Mathematics Subject Classification. Primary 20F65, 20 E08.

(C)2012 American Mathematical Society 3861

Reverts to public domain 28 years from publication 
scale' version of topological rather than geometric notions (see for example [9], 4]). Asymptotic topology notions are invariant under quasi-isometries (and one can see this as a large scale version of the fact that topological notions are invariant under isometries).

Let $K \geq 1, L \geq 0$ be constants. A $(K, L)$-quasi-isometry between two metric spaces $X, Y$ is a map $f: X \rightarrow Y$ such that the following two properties are satisfied:

1) $\frac{1}{K} d(x, y)-L \leq d(f(x), f(y)) \leq K d(x, y)+L$ for all $x, y \in X$.

2) For every $y \in Y$ there is an $x \in X$ such that $d(y, f(x)) \leq K$.

We will usually simply say quasi-isometry instead of $(K, L)$-quasi-isometry. Two metric spaces $X, Y$ are called quasi-isometric if there is a quasi-isometry $f: X \rightarrow Y$.

A geodesic metric space is a metric space in which any two points $x, y$ are joined by a path of length $d(x, y)$. One turns a connected graph into a geodesic metric space by giving each edge length 1 .

We call a map $f: X \rightarrow Y$ between metric spaces a uniform embedding (see [7]) if the following two conditions are satisfied:

1) There are $K, L$ such that for all $x, y \in X$ we have

$$
d(f b(x), f(y))<K(d(x, y))+L .
$$

2) For every $E>0$ there is $D>0$ such that $\operatorname{diam}(A)<E \Rightarrow \operatorname{diam}\left(f^{-1} A\right)<D$. If $f$ satisfies only condition 1 ) above we say that $f$ is a coarse lipschitz map. We remark that a coarse lipschitz map is a uniform embedding if there is a function $h: \mathbb{R}^{+} \rightarrow \mathbb{R}^{+}$such that for all $A \subset Y$, $\operatorname{diam}\left(f^{-1} A\right) \leq h(\operatorname{diam}(A))$. We then say that the distortion of $f$ is bounded by $h$. We remark that $f$ is a quasi-isometric embedding if its distortion is bounded by a linear function.

The following lemma ([7]) gives a simple characterization of uniform embeddings which will be used in the sequel.

Lemma 2.1. Let $X, Y$ be geodesic metric spaces. $f: X \rightarrow Y$ is a uniform embedding if and only if the following condition is satisfied: For any two sequences $\left(x_{n}\right),\left(y_{n}\right)$ of $X, d\left(x_{n}, y_{n}\right) \rightarrow \infty$ if and only if $d\left(f\left(x_{n}\right), f\left(y_{n}\right)\right) \rightarrow \infty$.

Proof. We first show that if the condition is satisfied, then $f$ is a uniform embedding. To see that $f$ satisfies 1 ) (i.e. is coarse lipschitz) we set

$$
K=\sup \{d(f(x), f(y)): d(x, y) \leq 1\} .
$$

Clearly $K<\infty$ by hypothesis. If $x, y$ are any two distinct points in $X$, we join them by a geodesic $\gamma$ and we pick consecutive points on $\gamma, x_{0}=x, x_{1}, \ldots, x_{n}=y$ so that $d\left(x_{i-1}, x_{i}\right)=1$ if $i<n$ and $d\left(x_{n-1}, x_{n}\right) \leq 1$. It follows that

$$
d(f(x), f(y)) \leq K n \leq K d(x, y)+K .
$$

Let $E>0$ be given. We set

$$
D=\sup \{d(x, y): d(f(x), f(y)) \leq E\} .
$$

Clearly $D<\infty$. This shows that $f$ satisfies 2$)$. Conversely now, assume that $f$ is a uniform embedding. If $d\left(f\left(x_{n}\right), f\left(y_{n}\right)\right) \rightarrow \infty$, since $d\left(f\left(x_{n}\right), f\left(y_{n}\right)\right)<$ $K\left(d\left(x_{n}, y_{n}\right)\right)+L$, it follows that $d\left(x_{n}, y_{n}\right) \rightarrow \infty$. Assume that $d\left(x_{n}, y_{n}\right) \rightarrow \infty$. If $d\left(f\left(x_{n}\right), f\left(y_{n}\right)\right)$ does not tend to infinity, then there is some $E>0$ and subsequences $x_{n_{k}}, y_{n_{k}}$ so that for all $k$,

$$
d\left(f\left(x_{n_{k}}\right), f\left(y_{n_{k}}\right)\right)<E .
$$


It follows by 2$)$ that there is some $D>0$ so that for all $k, d\left(x_{n_{k}}, y_{n_{k}}\right)<D$, which is a contradiction.

If $K \subset X$ and $R>0$ we say that a component of $X-N_{R}(K)$ is deep if it is not contained in $N_{R_{1}}(K)$ for any $R_{1}>0$. We say that $K$ coarsely separates $X$ if there is an $R>0$ such that $X-N_{R}(K)$ has at least two deep components. For example $X$ has more than one end if and only if $X$ is coarsely separated by a point.

We note that this asymptotic notion of separation was used, for example, to formulate a large scale version of Jordan's curve theorem for the plane (see [9], [5]).

Let $(X, d)$ be a geodesic metric space. If $R \subset X$ is a path connected subset of $X$, we denote the path metric of $R$ by $d_{R}$. We say that $R$ is a quasi-line if $\left(R, d_{R}\right)$ is quasi-isometric to $\mathbb{R}$ and the inclusion map $i:\left(R, d_{R}\right) \rightarrow(X, d)$ is a uniform embedding. We say that $R$ is a quasi-ray if $\left(R, d_{R}\right)$ is quasi-isometric to $\mathbb{R}^{+}$and the inclusion map $i:\left(R, d_{R}\right) \rightarrow(X, d)$ is a uniform embedding.

Kleiner has suggested the following asymptotic way to generalize the topological notion of a separating simple closed curve. Let $X$ be a geodesic metric space. Consider a sequence of simple closed curves $C_{n}$ in $X$ and denote by $d_{n}$ the path metric on $C_{n}$. We say that $C_{n}$ is a sequence of quasi-circles if diam $C_{n}$ tends to infinity and there is a distortion function $h: \mathbb{R}^{+} \rightarrow \mathbb{R}^{+}$such that

$$
d_{n}(a, b) \leq h(d(a, b))
$$

for any $a, b$ lying on any $C_{n}$.

We say that a sequence of quasi-circles $C_{n}$ coarsely separates $X$ if there is a $K$ (independent of $n$ ) such that $N_{K}\left(C_{n}\right)$ separates $X$ and at least two components of $X-N_{K}\left(C_{n}\right)$ are not contained in $N_{n}\left(C_{n}\right)$.
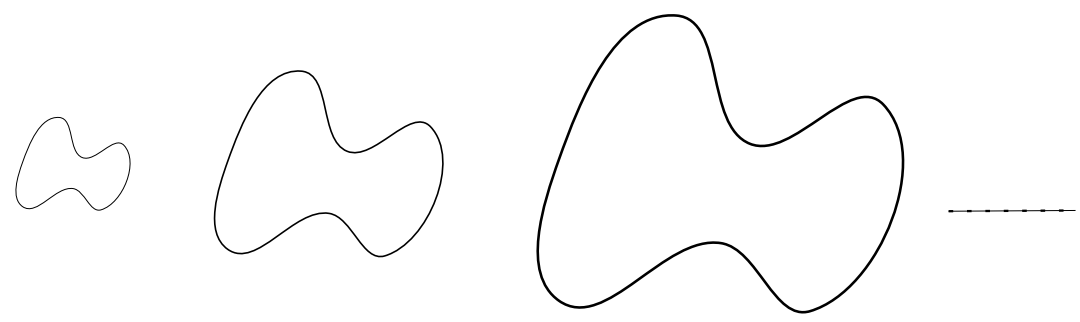

Figure 1. A sequence of quasi-circles.

We may similarly define a sequence of quasi-intervals $I_{n}$ which coarsely separates $X$ by taking $I_{n}=\left[a_{n}, b_{n}\right]$ to be simple paths, rather than simple closed curves.

2.2. Asymptotic topology and groups. We remark that if $H$ is a finitely generated subgroup of a finitely generated group $G$, then the embedding $H \hookrightarrow G$ is a uniform embedding (where we consider $H, G$ equipped with their word metrics).

Coarse separation is related to group splittings. We have the following easy lemma:

Lemma 2.2. If a finitely generated group $G$ splits over a finitely generated group $H$, then $H$ coarsely separates the Cayley graph of $G$.

Proof. If $G=A *_{H} B$ or $G=A *_{H}$ we may pick a finite generating set $S$ of $G$ so that, in the first case, all generators lie in $A \cup B$ or, in the second case, the generators 
are given by the stable letter $t$ and a finite set of elements of $A$. To see this take any finite set of generators of $G, S^{\prime}$ and write each element of $S^{\prime}$ in normal form with respect to the amalgam or the HNN-extension. Now take as a new generating set $S$ of $G$ the set of all elements of $A, B$ (and $t$ in the HNN-extension case) that appear in these normal form expressions. Let $\Gamma$ be the Cayley graph of $G$ with respect to $S$ and let $T$ be the Bass-Serre tree of $G$ for the splitting $G=A *_{H} B$ or $G=A *_{H}$. We consider the barycentric subdivisions $\Gamma^{\prime}$ of $\Gamma$ and $T^{\prime}$ of $T$. We now define a simplicial map $p: \Gamma^{\prime} \rightarrow T^{\prime}$. Let $e$ be the edge of $T$ with stabilizer $H$. Let $v$ be the midpoint of $e$, so $v$ is a vertex of $T^{\prime}$. We recall that the vertices of $\Gamma$ are the elements of $G$. If $g$ is a vertex of $\Gamma$ define $p(g)=g v$. If $(g, g s)$ is an edge of $\Gamma$ (so $g \in G, s \in S$ ), then $d(s v, v)$ is either 2 or 0 . So $d(g v, g s v)$ is 2 or 0 , and we can extend $p$ to $(g, g s)$ either by mapping it to the 2 consecutive edges joining $g v, g s v$ or by collapsing it to the vertex $g v$. This shows that the map $p$ can be extended from the set of vertices of $\Gamma$ to a simplicial map $p: \Gamma^{\prime} \rightarrow T^{\prime}$. Since the map $p^{\prime}$ is simplicial we have that $d(p(a), p(b)) \leq d(a, b)$ for all vertices of $\Gamma$. Further, $p$ is clearly onto. By our choice of $v, p^{-1}(v)=H$. Let $n \in \mathbb{N}$ and let $v_{1}, v_{2}$ be vertices of $T^{\prime}-T$ lying in distinct connected components of $T^{\prime}-v$ such that $d\left(v_{1}, v\right) \geq n, d\left(v_{2}, v\right) \geq n$. Let $g_{1} \in p^{-1}\left(v_{1}\right), g_{2} \in p^{-1}\left(v_{2}\right)$. Then if $\alpha$ is a path in $\Gamma^{\prime}$ joining $g_{1}$ to $g_{2}, v$ lies in $p(\alpha)$. It follows that $\alpha$ interects $p^{-1}(v)=H$. Further, if $h$ is the first vertex of $\alpha$ lying in $H$ and $\alpha_{1}=\left[g_{1}, h\right]$ the subpath of $\alpha$ with endpoints $g_{1}, h$, then $p\left(\alpha_{1}\right)$ joins $v_{1}$ to $v$. It follows that length $\left(p\left(\alpha_{1}\right)\right) \geq n$. Since $p$ is distance nonincreasing we conclude that $d\left(g_{1}, H\right) \geq n$. Similarly $d\left(g_{2}, H\right) \geq n$. Since this is true for any $n$ we conclude that $H$ coarsely separates $\Gamma$.

In the language of asymptotic topology Stallings' ends theorem ([14) says that a finitely generated group $G$ splits over a finite group if and only if the Cayley graph of $G$ is coarsely separated by a point.

Clearly, if a finitely generated group splits over a 2-ended group, then a quasi-line coarsely separates the Cayley graph of $G$. It was shown in [12] that the converse also holds when $G$ is 1-ended, finitely presented and not virtually a surface group. We will see in the next section that this characterization does not generalize to finitely generated groups.

Geometric characterizations of surface groups among finitely presented groups or hyperbolic groups were used in [12 and [1] to show that splittings over 2-ended groups are invariant under quasi-isometries. Such a characterization was also a crucial ingredient in the work of Mess [11] on the Seifert conjecture. It follows from [2] that a sequence of quasi-circles coarsely separates the Cayley graph of a one-ended finitely presented group $G$ if and only if $G$ is virtually a surface group. Kleiner asked whether this characterization of virtually surface groups extends to all finitely generated groups. We show in the next section that the Cayley graph of the lamplighter group $L$ is coarsely separated by quasi-circles, showing that the answer to Kleiner's question is negative.

\section{The ASYmptotic TOPOLOGY OF THE LAMPLighter GROUP}

The lamplighter group $L$ is defined as

$$
L=\left(\bigoplus_{\mathbb{Z}} \mathbb{Z}_{2}\right) \rtimes \mathbb{Z}
$$


where $\mathbb{Z}$ acts on the infinite direct sum $\bigoplus_{\mathbb{Z}} \mathbb{Z}_{2}$ by sending the generator of the $i$-th factor, $x_{i}$, to the generator of the next factor, $x_{i+1}(i \in \mathbb{Z})$. It is easy to see that $L$ is in fact finitely generated and is given by the presentation

$$
L=\left\langle a, t \mid a^{2},\left[t^{i} a t^{-i}, t^{j} a t^{-j}\right], i, j \in \mathbb{Z}\right\rangle .
$$

We will denote by $X$ the Cayley graph of $L$ with respect to the generators $a, t$.

3.1. Elements of the lamplighter group and metric properties. It is convenient to represent elements of $L$ geometrically. We briefly explain how this is done. We see $L$ as a semi-direct product

$$
L=\left(\bigoplus_{\mathbb{Z}} \mathbb{Z}_{2}\right) \rtimes \mathbb{Z}
$$

so an element $g$ of $L$ is of the form $g=(h, k)$, where $h \in \oplus_{\mathbb{Z}} \mathbb{Z}_{2}, k \in \mathbb{Z}$. Now since $h$ is an element of a direct sum of $\mathbb{Z}_{2}$ 's it can be seen as a bi-infinite string of 0 's and 1's with at most finitely many 1's. One thinks of these strings of 0 and 1 's as lamps at the integers on the real line. A 0 indicates a lamp which is off, while a 1 indicates a lamp which is lit. We encode $g=(h, k)$ by the infinite string of lamps corresponding to $h$ with a cursor at position $k$. If we denote by $e$ the identity element of $\bigoplus_{\mathbb{Z}} \mathbb{Z}_{2}$ and by $x_{0}$ the element of $\bigoplus_{\mathbb{Z}} \mathbb{Z}_{2}$ given by 1 at position 0 and 0 everywhere else, then clearly $t=(e, 1)$ and $a=\left(x_{0}, 0\right)$ generate $L$. By the representation of $L$ as a semi-direct product $L=\left(\bigoplus_{\mathbb{Z}} \mathbb{Z}_{2}\right) \rtimes \mathbb{Z}$, one obtains the presentation

$$
L=\left\langle a, t \mid a^{2},\left[t^{i} a t^{-i}, t^{j} a t^{-j}\right], i, j \in \mathbb{Z}\right\rangle
$$

for the generators $a, t$.

We now explain how the generators $a, t$ act on the geometric representation of the elements of the lamplighter group. Clearly

$$
(h, k) \cdot t=(h, k) \cdot(e, 1)=(h, k+1) .
$$

So multiplication by $t$ has the effect of moving the cursor by 1 to the right. Also

$$
(h, k) \cdot a=(h, k) \cdot\left(x_{0}, 0\right)=\left(h \cdot x_{k}, k\right),
$$

where $x_{k}$ is the element of $\bigoplus_{\mathbb{Z}} \mathbb{Z}_{2}$ given by 1 at the $k$-th position and 0 everywhere else. So the $t$ generator corresponds to a move of the cursor to the right, while the $a$ generator corresponds to switching a lamp at the position of the cursor.

One calls the cursor a 'lamplighter'. A word on the $a, t$ generators corresponds to a sequence of 'moves' of the lamplighter, and the $t$ 's correspond to a change of position by 1 while the $a$ 's to switching the lamp at the position of the lamplighter.

If we think that it takes 1 unit of time for the lamplighter to move from position $n$ to position $n+1$ (or $n-1$ ) and 1 unit of time to turn the switch on or off, then the distance between the identity configuration (lamps off, lamplighter at 0) to a given configuration is given by the time it takes the lamplighter to turn on the lamps and to move to the position of the given configuration. For a more detailed exposition of normal forms and the geometric representation of the elements of $L$ we refer to [3].

We now introduce some notation that will be useful in the sequel.

We associate to each configuration $c$ of 0,1 's on $\mathbb{Z}$ a binary integer $c^{+}$, where $c^{+}$is the binary number that we obtain from the digits of $c$ which are right from 0 starting at 0 (where we read the number in reverse, from right to left). Similarly 


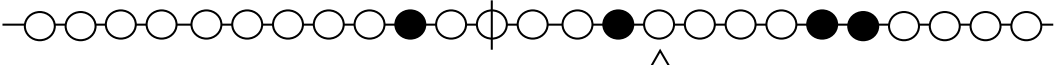

Figure 2. Here we give an example of a geometric representation of an element of $L$. The vertical line stands for the origin, lamps which are on are filled-in circles and lamps which are off are circles with no filling. The lamplighter position is indicated by a triangle. The lamplighter in this example is at position 4 .

we associate to $c$ the binary number $c^{-}$, which is the number which we read from $c$ if we start at -1 and move left. So, for example, if $c$ is the configuration

$$
c=\ldots 0,0,1,0, S 0,1,1,0,0, \ldots,
$$

where we denote by $S$ the origin, then $c^{+}=011=0 \cdot 1+1 \cdot 2+1 \cdot 2^{2}=6$ and $c^{-}=10=0 \cdot 1+1 \cdot 2=2$ (see Figure 2). If $c$ is a configuration we denote by $c(k)$ the digit at position $k$. So for instance in the example above we have $c(0)=0, c(-2)=1, c(1)=1$. With this notation we have

$$
c^{+}=\sum_{k=0}^{\infty} c(k) 2^{k}, \quad c^{-}=\sum_{k=-1}^{-\infty} c(k) 2^{-k-1} .
$$

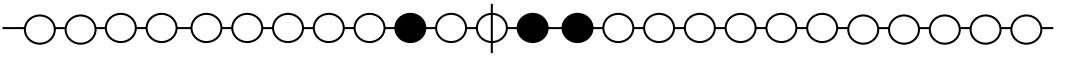

FiguRE 3. In this configuration $c^{+}=011=0 \cdot 1+1 \cdot 2+1 \cdot 2^{2}=6$ and $c^{-}=10=0 \cdot 1+1 \cdot 2=2$. The position of the lamplighter does not affect $c^{+}$and $c^{-}$.

3.2. A separating quasi-line in the lamplighter group. We now show that there is a quasi-ray which coarsely separates the Cayley graph of $L$ (problem 4.2 in [13). This contrasts with the case of finitely presented groups where it is impossible for a quasi-ray to coarsely separate the Cayley graph of a 1-ended group (this can be shown using the results and methods of [12]).

Before proceeding to the formal proof we explain the idea of the construction of the separating quasi-ray. Consider the set of all configurations $c$ such that $c^{-}$is 0 and the lamplighter is at position 0 . Let's denote by $A$ the subset of $L$ corresponding to these configurations. If $X$ is the Cayley graph of $L$, it is not very hard to see that $A$ separates $X$. Indeed, let $x_{n}$ be the configuration with 0 everywhere and the lamplighter at $n$ and let $y_{n}$ be the configuration with 0 everywhere and the lamplighter at $-n$. Then to move from $x_{n}$ to $y_{n}$ the lamplighter has to pass by position 0 . However, no matter how the lamplighter moves, starting from $x_{n}$, the first time that he reaches 0 the resulting configuration is an element of $A$. This shows that $A$ separates $x_{n}$ from $y_{n}$.

Now the idea is to create a quasi-ray that contains $A$. The main technical issue while doing this is to control distortion. Of course there are many ways to do this, so our construction is, in a sense, quite arbitrary.

Theorem 3.1. There is a quasi-ray $N$ which coarsely separates the Cayley graph of $L, X$. 

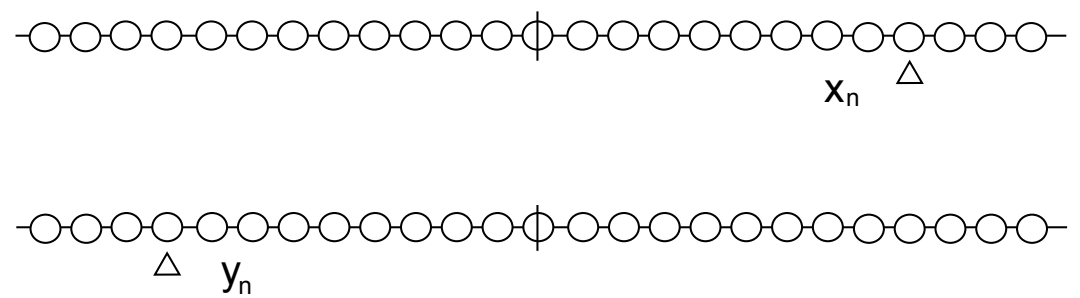

Figure 4. $A$ separates $x_{n}, y_{n}$.

Proof. We describe $N$ using the geometric representation of the elements of $L$ described earlier. We split the proof into 3 parts. In the first part we define $N$. In the second part we show that $N$ is uniformly embedded. In the third part we show that it coarsely separates $X$.

(1) $N$ will be defined as a graph isomorphic to a half-line given by a sequence of vertices of $X$. Using our geometric representation we may define $N$ as an infinite 'trip' of the lamplighter which moves on the line, turning switches on and off. It will be convenient to describe this trip in stages.

The first vertex of $N$ is the identity configuration: the lamplighter at 0 and all lamps at the 0 state (off). This is the stage 0 of the trip. We now describe how the lamplighter moves. At stage $n(n \in \mathbb{N})$ we are at a vertex given by the configuration $c_{n}$ where the lamplighter is at the origin, $c_{n}^{+}$is the number $n$ written in binary and there are only 0's left of the lamplighter. Now to go from the stage $n$ to the stage $n+1$ the lamplighter does the following: If $c_{n}(0)=0$, then he simply switches 0 to 1 . The configuration obtained in this way is the configuration of stage $n+1$. If $c_{n}(i)=1$ for all $i, 0 \leq i \leq k-1$ and $c_{n}(k)=0$, then the lamplighter moves left to $-k$, switches it to 1 and moves back to the origin turning the switch at position $-k+r$ so that it is the same as the switch at position $k+r$ (for all $r \leq k-1)$. We denote the configuration obtained after this by $c_{n 0}$. After that the lamplighter moves to the right, switching as he moves all lamps that are on to off until he reaches the $k$-th lamp which is off and switches it on. If we denote by $c_{n 1}$ the configuration that results from this, it is easy to see that

$$
c_{n 1}^{+}=n+1
$$

After that the lamplighter moves left again until he reaches the origin, after the origin he goes left, turning off all lamps which are on, and after turning off the last lamp which is on, at position $-k$, returns to the origin. In this way we move from configuration $c_{n}$ to $c_{n+1}$. It is clear that $N$ does not meet the same configuration twice, so $N$ is indeed a half-line.

(2) We now show that $N$ is uniformly embedded. Let's denote by $N(i)$ the $i$-th vertex of $N$.

It suffices to show the following: If $a=N(i), b=N(j)(i<j)$ and $d(a, b) \leq M$, then $j-i$ is bounded by a constant $D=D(M)$ which depends only on $M$. Let's denote by $l(a), l(b)$ the lamplighter positions for $a, b$, respectively. We remark that

$$
|l(a)-l(b)| \leq d(a, b) \leq M .
$$


We now distinguish some cases:

Case 1: $l(a), l(b) \in[-M, M]$. Then the configurations of $a, b$ are identical in $[2 M, \infty]$. This implies that when we move from $N(i)$ to $N(j)$ the lamplighter moves in the interval $[-2 M, 2 M]$. But then there is a bounded number of new configurations that can be created so $j-i$ is bounded in this case by a constant $D_{1}=D_{1}(M)$.

Case 2: At least one of $l(a), l(b)$ lies in $[M+1, \infty)$. Then both $l(a), l(b)$ lie in $[1, \infty)$. It is clear that if there is some $n$ so that both $a, b$ are configurations that appear as we move from $c_{n}$ to $c_{n+1}$, then $j-i \leq M$. We therefore assume that $a$ is a configuration that appears in the stage $c_{k}$ to $c_{k+1}$, while $b$ is a configuration that appears in the stage $c_{n}$ to $c_{n+1}$ for some $n>k$. Clearly $|l(a)-l(b)| \leq M$. We remark that since $l(a), l(b)>0, a$ is a configuration that appears at the stage $c_{k 0}$ to $c_{k}$ and $b$ is a configuration that appears at the stage $c_{n 0}$ to $c_{n}$. We now recall that as the lamplighter moves from $c_{k 0}$ to $c_{k 1}$ he erases a string of consecutive 1's. Let's say that the initial string of 1's of $c_{k}$ consists of $d_{k} 1$ 's and that the initial string of 1's of $c_{n}$ consists of $d_{n} 1$ 's. By the definition of $c_{k 0}, c_{n 0}$ the lamps at $-d_{k}-1,-d_{n}-1$ are respectively on for these two configurations. Recall that, by the definition of $N$, to move from configuration, say $c_{k}$ to $c_{k 0}$, the lamplighter moves left to $-d_{k}-1$ and turns the lamp on before returning to the origin. If $d_{k} \neq d_{n}$, then $d(a, b)>M$. This is because, by the way they were defined, $c_{k 0}^{-}$and $c_{n 0}^{-}$differ at the position $\min \left(-d_{k}-1,-d_{n}-1\right)$ (and it takes the lamplighter more than $M$ steps to change this, since one of $l(a), l(b)$ is in $[M+1, \infty))$.

We assume therefore that $d_{k}=d_{n}=d>M$. Since $d(a, b) \leq M$ we have that $c_{k 0}^{-}=c_{n 0}^{-}$. This however implies that the configurations $c_{k}, c_{n}$ are identical in all positions in $[0,2 d]$. Indeed recall that, by the definition of $N$, as the lamplighter moves from $c_{k}$ to $c_{k 0}$ he goes to $-d_{k}-1$, switches the lamp on, and as he returns to 0 turns lamps on in the same way as in the interval $[d, 2 d]$. In other words the configuration $c_{k 0}^{-}$in the interval $[-d-1,-1]$ is identical to the part of configuration $c_{k}$ in the interval $[d, 2 d]$. Since $d(a, b) \leq M$ and the lamplighter for $a, b$ lies in the interval $[0, d]$, we also have that $c_{k}, c_{n}$ are identical in $[d+M, \infty)$. But $d>M$, so $c_{n}=c_{k}$, which is a contradiction since we assumed that $n>k$.

Case 3: At least one of $l(a), l(b)$ lies in $(\infty,-M-1]$. As in Case 2 we see that $a$ is a configuration that appears in the stage $c_{k}$ to $c_{k+1}$, while $b$ is a configuration that appears in the stage $c_{n}$ to $c_{n+1}$ for some $n>k$. However, since $k \neq n$ we have that $c_{k}^{+} \neq c_{n}^{+}$. On the other hand one of $l(a), l(b)$ lies in $(\infty,-M-1]$. This implies that $d(a, b)>M$.

This completes the proof that $N$ is uniformly embedded.

(3) We finally show that $N$ coarsely separates $X$. To show this it is enough to show that there are sequences of vertices $x_{n}, y_{n}$ in $X$ such that

$$
d\left(x_{n}, N\right) \geq n, d\left(y_{n}, N\right) \geq n
$$

and $x_{n}, y_{n}$ lie in distinct components of $X-N$. We define $x_{n}$ to be the following configuration: $x_{n}^{+}$consists of $2 n$ consecutive 1's, $x_{n}^{-}$consists of 0's and the lamplighter is at the position $n . y_{n}$ is the configuration with 0's everywhere and the lamplighter at $-n$. It is now clear that to move from $x_{n}$ to $N$ the lamplighter has to erase all 1's at the interval $[0, n]$, so $d\left(x_{n}, N\right) \geq n$. Similarly, to move from $y_{n}$ to $N$ the lamplighter has to move from position $-n$ to 0 , so $d\left(y_{n}, N\right) \geq n$. 
We now show that $x_{n}, y_{n}$ are separated by $N$. To move from $x_{n}$ to $y_{n}$ the lamplighter has to go through 0 . But no matter how the lamplighter switches lamps on and off before reaching 0 , when the lamplighter moves to 0 for the first time we are exactly at the beginning of stage $c_{k}$, where $k$ is the integer whose binary expression we see on the right side. This is because we assume that the 'trip' of the lamplighter starts at $x_{n}$, so all lamps at the left side of 0 are off. So the first time the lamplighter arrives at 0 all lamps at the left side of 0 are off. We conclude that any path joining $x_{n}, y_{n}$ in $X$ intersects $N$.

Some examples. As the definition of $N$ in the previous proof is quite ad hoc, we now give some examples to illustrate why more naive ways to define $N$ wouldn't produce a uniformly embedded set. Let's say that we define $N$ to be the path obtained as the lamplighter successively writes the numbers $1,2,3, \ldots$ in binary (that is, $N$ is defined as before, but the lamplighter never goes left of position 0 ; in other words we eliminate the stage $c_{n 0}$ to $c_{n 1}$ of our definition). Then when the lamplighter writes the number $2^{m}$ we have the following configuration: Lamplighter at $m$ and 0 's at all positions except at $m$ where we have 1 . Let's call the vertex of $X$ represented by this configuration $v(m)$.
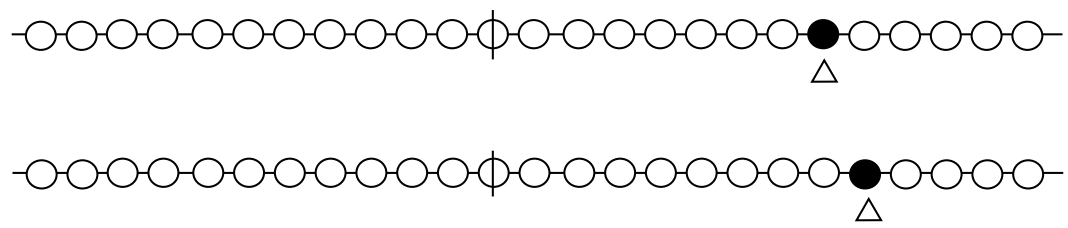

FiguRE 5. Far away in $N$ but close in the Cayley graph.

We then see that $v(m)$ and $v(m+1)$ are at distance 3 in $X$ while to move from $v(m)$ to $v(m+1)$ takes more than $2^{m}$ steps in $N$. This produces unbounded distortion. To avoid this problem we introduced the stage $c_{n 0}$ to $c_{n 1}$ in our definition of $N$ which gives a 'marker' for the stage we are in. A more naive 'marker' wouldn't work either. Say for example that in the stage $c_{n 0}$ to $c_{n 1}$ the lamplighter just moves left and only turns on the lamp at position $-k$ and no other lamp on his way back to 0 . Then again we will have unbounded distortion. Indeed consider the steps of the procedure in which the lamplighter writes the numbers $2^{n+2}+2^{n}$ and $2^{n+3}+2^{n}$. The 'markers' for these two configurations are the same (a lamp lit at position $-n$ ). However, these configurations correspond to points that are close in $X$ but far away in $N$.
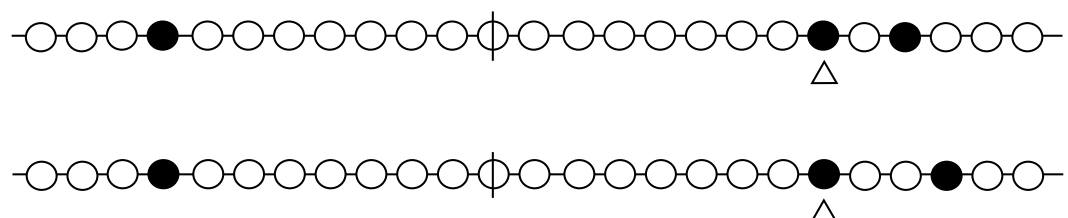

Figure 6 . The more naive 'marker' depicted here does not suffice to bound distortion. 
It is not very hard to see that $X$ is also separated by a quasi-line. Indeed we define a quasi-line $R$ by $R(i)=N(i)$ for all $i \in \mathbb{N}$ and we define $R(-i)$ to be the lamplighter at $-i$ and the lamps in $[-i, 0]$ are on (and all other lamps off). One easily sees that $R$ is uniformly embedded in $X$. It is clear that if we take $x_{n}, y_{n}$ as in the previous proof, we have that $d\left(x_{n}, R\right) \geq n, d\left(y_{n}, R\right) \geq n$ so that $R$ coarsely separates $X$. That is, we have the following:

Corollary 3.2. There is a quasi-line $R$ which coarsely separates the Cayley graph of $L, X$.

Proposition 3.3. $L$ is one-ended and does not split over a 2-ended group.

Proof. By using Stallings' theorem to show that $L$ is one-ended, it suffices to show that $L$ does not split over a finite group. Assume that $L$ splits over a finite or 2-ended subgroup $C$ and consider the Bass-Serre tree $T$ of this splitting. Then if we write $L$ as a semi-direct product

$$
L=\left(\bigoplus_{\mathbb{Z}} \mathbb{Z}_{2}\right) \rtimes \mathbb{Z}
$$

we claim that the direct sum $\bigoplus_{\mathbb{Z}} \mathbb{Z}_{2}$ fixes some vertex $v$ of the tree $T$. Indeed, a finite group acting on a tree without inversions fixes a vertex of the tree. $\bigoplus_{\mathbb{Z}} \mathbb{Z}_{2}$ is an ascending union of the finite groups

$$
F_{n}=\left\langle x_{i}: i=-n, \ldots, n\right\rangle,
$$

where $x_{i}$ are the generators of the $\mathbb{Z}_{2}$ summands. If $\bigoplus_{\mathbb{Z}} \mathbb{Z}_{2}$ does not fix a vertex of $T$, for arbitrarily big $n F_{n}$ fixes a vertex $v_{n}$, which is not fixed by $F_{n-1}$. If $u_{n}$ is a vertex fixed by $F_{n-1}$, then the path joining $u_{n}$ and $v_{n}$ is fixed by $F_{n-1}$. This however contradicts our assumption that the edge stabilizers of $T$ are all conjugate to a fixed finite or 2-ended group and hence have bounded torsion.

If the generator $t$ of $\mathbb{Z}$ sends $v$ to $t(v) \neq v$, then, since $t x_{i} t^{-1}=x_{i+1}$ (where $x_{i}$ is the generator of the $i$-th $\mathbb{Z}_{2}$ factor) we have that $\bigoplus_{\mathbb{Z}} \mathbb{Z}_{2}$ also fixes $t(v)$, so there is an edge fixed by $\bigoplus_{\mathbb{Z}} \mathbb{Z}_{2}$. Clearly, if $t(v)=v$, then $L$ fixes a vertex of the tree. In both cases we see that there is no action with finite or 2-ended edge stabilizers.

3.3. Quasi-circles. We now show that there is a sequence of quasi-circles that coarsely separates the Cayley graph, $X$ of $L$. In fact, we show that a sequence of quasi-intervals $I_{n}$ coarsely separates $X$. We then show that one can 'close up' these quasi-intervals to quasi-circles that coarsely separate $X$.

The construction of the quasi-intervals is similar to the construction of $N$.

We fix $n \in \mathbb{N}$. We now define a simple path $I_{n}$ and a simple closed path $C_{n}$ such that there are $x_{n}, y_{n} \in X$ which are separated by $I_{n}\left(C_{n}\right)$ and such that

$$
d\left(x_{n}, I_{n}\right)>n, d\left(y_{n}, I_{n}\right)>n, d\left(x_{n}, C_{n}\right)>n, d\left(y_{n}, C_{n}\right)>n .
$$

The interval $I_{n}$ is defined as follows: We think of $I_{n}$ as a path $I_{n}(i), i \in \mathbb{N}$. If $N$ is the quasi-ray defined in the previous section, we set $I_{n}(i)=N(i)$ for all $i$ until we reach the configuration consisting of 1 's in positions $0,1, \ldots, 2 n$, the lamplighter at the origin and 0 everywhere else. After this the lamplighter moves from position 0 to position $2 n$ switching off the lamps as he goes. Let's say that $I_{n}(k)$ is the vertex of $X$ that we get when the lamplighter reaches $2 n$.

When the lamplighter reaches $2 n$ he repeats the steps exactly as he did at the beginning, except that he walks in the reverse direction. More formally, let's denote by $q(i)$ the walk of the lamplighter, where for any $i, q(i)$ is either a move 
by one to the right, denoted by +1 , or a move to the left, denoted by -1 , or a change of switch denoted by 0 . Then if $q(k)$ is the lamplighter at $I_{n}(k)$ we define $q(k+i)=-q(i)$. The lamplighter continues until he reaches the following configuration: Lamplighter at $2 n$ and configuration consisting of 1 's in positions $0,1, \ldots, 2 n$, and 0 everywhere else. We will now show that the sequence $I_{n}$ is a sequence of quasi-intervals. In order to analyse distortion we think of $I_{n}$ as constructed in 3 stages. So we write

$$
I_{n}=I_{n 1} \cup I_{n 2} \cup I_{n 3},
$$

where $I_{n 1}, I_{n 2}, I_{n 3}$ are defined as follows: $I_{n 1}$ is the initial path, so $I_{n 1}=I_{n} \cap N$. $I_{n 3}$ is the last stage where the lamplighter moves exactly as in $I_{n 1}$ but in the reverse direction and starting from $2 n$ rather than 0 . Finally $I_{n 2}$ is the path of $I_{n}$ joining $I_{n 1}, I_{n 3}$. In order to show that $I_{n}$ is a sequence of quasi-intervals, it is enough to show that there are no sequences $a_{n}, b_{n} \in I_{n}$ such that

$$
a_{n}=I_{n}\left(i_{n}\right), b_{n}=I_{n}\left(j_{n}\right)
$$

with

$$
\limsup _{n \rightarrow \infty}\left|i_{n}-j_{n}\right|=\infty
$$

and $d\left(a_{n}, b_{n}\right)=M$ for some fixed $M \in \mathbb{N}$. Since $N$ is uniformly embedded in $X$ it is clear that it is not possible that for infinitely many $n, a_{n}, b_{n}$ are contained both in $I_{n 1}$ or in $I_{n 3}$. Since $I_{n 2}$ is a geodesic path it follows similarly that $a_{n}, b_{n}$ cannot both be contained in $I_{n 2}$ for infinitely many $n$. Finally, since $d\left(I_{n 1}, I_{n 3}\right)>n$ it is not possible that for infinitely many $n$ one of the $a_{n}, b_{n}$ is contained in $I_{n 1}$ and the other in $I_{n 3}$. So at least one of the following two holds:

Case 1. For infinitely many $n, a_{n} \in I_{n 1}, b_{n} \in I_{n 2}$.

Case 2. For infinitely many $n, a_{n} \in I_{n 2}, b_{n} \in I_{n 3}$.

Assume now that we are in Case 1 and consider the geometric representation of $a_{n}, b_{n}$. If the lamplighter for $b_{n}$ is in $[0, M]$, then, since $d\left(a_{n}, b_{n}\right) \leq M$, the configurations for $a_{n}, b_{n}$ are identical in $[2 M, \infty)$. But, by the definition of $I_{n}$, this implies that $j_{n}-i_{n}$ is bounded by some constant $D(M)$ depending only on $M$. If the lamplighter for $b_{n}$ lies in $[M+1,2 n)$, we remark that either the lamplighter for $a_{n}$ is at 0 , so $d\left(a_{n}, b_{n}\right)>M$, or the configuration of $a_{n}$ has some lamp on in $[-2 n, 1]$. However, in this case, $d\left(a_{n}, b_{n}\right)>M$, too.

We assume now that we are in Case 2. If the lamplighter for $a_{n}$ lies in $[0,2 n-$ $M-1]$, then clearly $d\left(a_{n}, b_{n}\right)>M$. If the lamplighter for $a_{n}$ lies in $[2 n-M, 2 n]$, then the configuration of $b_{n}$ has to be identical with the configuration of $a_{n}$ in $[0,2 n-M]$ (since $\left.d\left(a_{n}, b_{n}\right)=M\right)$. However, this implies that $j_{n}-i_{n}$ is smaller than a constant $D(M)$ that depends only on $M$. We see in both cases that it is impossible to have

$$
\limsup _{n \rightarrow \infty}\left|i_{n}-j_{n}\right|=\infty
$$

so $I_{n}$ is indeed a sequence of quasi-intervals.

We now show that there are $x_{n}, y_{n}$ in $X$ such that

$$
d\left(x_{n}, I_{n}\right) \geq n, d\left(y_{n}, I_{n}\right) \geq n
$$

and $x_{n}, y_{n}$ lie in distinct components of $X-I_{n}$. We take $x_{n}$ to be the following configuration: Lamplighter at $n, 1$ 's at the interval $[0,2 n]$ and 0 's everywhere else. We take $y_{n}$ to be the lamplighter at $-n$ and 0 everywhere. By the way they are 
defined $x_{n}, y_{n}$ satisfy $d\left(x_{n}, I_{n}\right) \geq n, d\left(y_{n}, I_{n}\right) \geq n$. We now remark that $I_{n 1}$ contains all the configurations of the following form: Lamplighter at 0, and 0's outside the interval $[0,2 n]$. Putting it differently, $I_{n 1}$ contains all possible configurations of 0,1 's in $[0,2 n]$ with the lamplighter at 0 and 0 's elsewhere. Similarly $I_{n 3}$ contains all possible configurations in $[0,2 n]$ with the lamplighter at $2 n$ and 0 's elsewhere. It is clear that to move from $x_{n}$ to $y_{n}$ the lamplighter has to pass through 0 . There are two cases.

Case 1. The lamplighter, while moving from $x_{n}$ to $y_{n}$, passes through position 0 before passing through position $2 n$ (of course the lamplighter might not pass through position $2 n$ at all; then we are also in this case). In this case the first time the lamplighter arrives at 0 , every lamp is off outside the interval $[0,2 n]$. So the lamplighter is at a point of $I_{n 1}$.

Case 2. The lamplighter, while moving from $x_{n}$ to $y_{n}$, passes through position $2 n$ before passing through position 0 . In this case the first time the lamplighter arrives at $2 n$, every lamp is off outside the interval $[0,2 n]$. So the lamplighter is at a point of $I_{n 3}$.

It follows that $I_{n 1} \cup I_{n 3}$ separates $x_{n}$ from $y_{n}$. This shows that the sequence of quasi-intervals $I_{n}$ coarsely separates $X$.

It is easy to define a sequence of quasi-circles $C_{n}$ which coarsely separates $X$, using the quasi-intervals $I_{n}$. Namely, we join the endpoint of $I_{n}$ to the initial point as follows: The lamplighter moves from $2 n$ back to 0 , turning off as he goes all lamps in the interval $[0,2 n]$. Let's call this last interval $I_{n 4}$. So

$$
C_{n}=I_{n 1} \cup I_{n 2} \cup I_{n 3} \cup I_{n 4} .
$$

It suffices to check that, given $M>0$, there are no sequences $a_{n}, b_{n} \in C_{n}$ with the following properties:

1) $d\left(a_{n}, b_{n}\right)=M$.

2) If $l_{n}$ is the length of the shortest path contained in $C_{n}$ joining $a_{n}, b_{n}$, then $l_{n} \rightarrow \infty$.

Since $I_{n}=I_{n 1} \cup I_{n 2} \cup I_{n 3}$ is uniformly embedded and since $I_{n 4}$ is a geodesic segment, it follows that, for infinitely many $n$, one of $a_{n}, b_{n}$ lies in $I_{n}$ and the other lies in $I_{n 4}$. However, one easily sees by the definition of $I_{n 4}$ that if $l_{n} \rightarrow \infty$, then $d\left(a_{n}, b_{n}\right) \rightarrow \infty$. It follows that the $C_{n}$ 's are uniformly embedded (i.e. it is a sequence of quasi-circles).

If we pick $x_{n}, y_{n}$ as before we see that

$$
d\left(x_{n}, C_{n}\right) \geq n, d\left(y_{n}, C_{n}\right) \geq n
$$

and $x_{n}, y_{n}$ are separated by $C_{n}$ since they are already separated by $I_{n} \subset C_{n}$.

\section{Discussion}

The idea behind the coarse separation properties of the lamplighter group comes from the fact that the asymptotic dimension of the lamplighter group is 1 ([6], [8]). We remark that if the asymptotic dimension of a finitely presented group is 1 , then the group is virtually free $([6])$. So one can think of the lamplighter group as a group theoretic (or 'asymptotic') analog of the Menger curve. As the topological dimension of the Menger curve is 1 , the Menger curve is separated by a totally disconnected set (if one uses the standard construction of the Menger curve drilling 
holes in a cube, a separating totally disconnected set is obtained by a plane cutting the cube in two halves). It turns out that one can find a simple path containing this totally disconnected separating subset. Our construction is in a way a large scale version of this: If we try to visualize the set $A$ defined before the proof of Theorem 3.1 we see that as we move further and further from the identity and we look at larger scales the points in $A$ are quite sparse. This is because even when two configurations are quite close the lamplighter has to move a large distance to transform one configuration to the other. Although we don't prove this here, one could state this property of $A$ formally by saying that $A$ is a set of asymptotic dimension 0 that separates $X$. In the proof of Theorem 3.1 we show that there is a quasi-ray that contains $A$.

This remark prompts us to reformulate some of the questions treated in this paper.

Question. Let $G$ be a one-ended finitely generated group and let $X$ be the Cayley graph of $G$. Assume that no subset of asymptotic dimension 0 of $X$ coarsely separates $X$. Is it true that no sequence of quasi-circles coarsely separates $X$ ? Is it true that no quasi-ray coarsely separates $X$ ?

\section{REFERENCES}

[1] B.H. Bowditch, Cut points and canonical splittings of hyperbolic groups, Acta Math. 180, No. 2, pp. 145-186 (1998). MR1638764 (99g:20069)

[2] B.H. Bowditch, Planar groups and the Seifert conjecture, Journal fur die reine und angewandte Mathematik (Crelle's Journal), Volume 2004, Issue 576, 11-62. MR2099199 (2005h:57033)

[3] S. Cleary, J. Taback, Dead end words in lamplighter groups and other wreath products, Quarterly Journal of Mathematics, Vol. 56, Number 2, June 2005, pp. 165-178. MR2143495 (2006h:20055)

[4] A. Dranishnikov, Asymptotic Topology, Russian Math. Surveys 55, (2000), no. 6, 71-116. MR1840358 (2002j:55002)

[5] B. Farb, R. Schwartz, The large-scale geometry of Hilbert modular groups, J. Diff. Geom., vol. 44 (1996) pp. 435-478. MR1431001 (98f:22018)

[6] T. Gentimis Asymptotic dimension of finitely presented groups, Proc. Amer. Math. Soc. 136 (2008), 4103-4110. MR2431020 (2009e:20095)

[7] M. Gromov, Asymptotic invariants of infinite groups, in Geometric group theory (G. Niblo, M. Roller, eds.), LMS Lecture Notes, vol. 182, Cambridge Univ. Press, 1993. MR 1253544 (95m:20041)

[8] T. Januszkiewicz, J. Swiatkowski, Filling invariants of systolic complexes and groups, Geometry and Topology 11 (2007), pp. 727-758. MR2302501 (2008d:20079)

[9] M. Kapovich, B. Kleiner, Coarse Alexander duality and duality groups, J. Differential Geom. 69, no. 2, 279-352, 2005. MR2168506 (2007c:57033)

[10] B. Kleiner, in preparation.

[11] G. Mess, The Seifert conjecture and groups which are coarse quasiisometric to planes preprint, 1990.

[12] P. Papasoglu, Quasi-isometry invariance of group splittings, Annals of Math. vol. 161, no. 2, pp. 759-830 (2005). MR2153400 (2006d:20076)

[13] P. Papasoglu, JSJ decompositions, in Problems in Geometric group theory, http:// aimath.org/pggt.

[14] J.R. Stallings, On torsion-free groups with infinitely many ends, Ann. of Math. 88, pp. 312334 (1968). MR0228573 (37:4153)

Mathematical Institute, University of Oxford, 24-29 St. Giles', Oxford, OX1 3LB, United Kingdom

E-mail address: papazoglou@maths.ox.ac.uk 